\title{
A Robotic Exoskeleton to Treat Crouch Gait from Cerebral Palsy: Initial Kinematic and Neuromuscular Evaluation*
}

\author{
Zachary F. Lerner, Diane L. Damiano, and Thomas C. Bulea, Member, IEEE
}

\begin{abstract}
A robotic exoskeleton was designed for individuals with crouch gait caused by cerebral palsy with the intent to supplement existing muscle function during walking. The aim of this study was to evaluate how powered knee extension assistance provided during stance and swing phases of the gait cycle affect knee kinematics, and knee flexor and extensor muscle activity. Muscle activity and kinematic data were collected from four individuals with crouch gait from cerebral palsy during their normal walking condition and while walking with the exoskeleton under stance, swing, and stance \& swing assistance. The exoskeleton was effective in reducing crouch by an average of $13.8^{\circ}$ in three of the four participants when assistance was provided during the stance phase; assistance during the swing phase alone was ineffective. Peak knee extensor activity was maintained for all of the conditions during the stance and swing phases. Integrated (i.e. area under the curve) knee extensor activity decreased in two of the subjects indicating a more well-modulated activation pattern. Modest increases in peak and integrated antagonist knee flexor activity were exhibited in all participants; the subject without kinematic improvement had the greatest increase. While the exoskeleton was well tolerated, additional training with a focus on reducing knee flexor activity may lead to further improvements in crouch gait reduction.
\end{abstract}

\section{INTRODUCTION}

Cerebral palsy (CP) is a childhood-onset, non-progressive motor control disorder that is accompanied by pathological gait patterns in many affected individuals [1]. One of the most common impaired walking patterns is crouch gait which is characterized by excessive knee flexion [2]. In addition to increasing the metabolic cost of walking, crouch gait can lead to knee pain and degeneration [3-5]. Established treatment strategies, including orthopaedic surgery [6], targeted muscle strengthening [7], and orthotic bracing [8], can alleviate crouch but do not result in complete correction and long-term improvements remain limited. There is a need for new or improved strategies that can preserve or augment function in order to prevent ambulatory decline in adulthood [9].

During crouch gait, the knee is excessively flexed at initial foot contact and remains flexed throughout stance phase [2]. Robotic exoskeletons have the potential to treat crouch in CP by providing powered knee extension assistance either during the swing phase, acting to extend the knee prior to initial contact, during the stance phase, acting to support the body, or both.

* This work was supported by the Intramural Research Program at the National Institutes of Health (protocol \# 13-CC-0210).

Z.F. Lerner, D.L. Damiano, and T.C. Bulea are with the Functional and Applied Biomechanics Section, Rehabilitation Medicine Department, Clinical Center, National Institutes of Health, Bethesda, MD, 20892 USA (phone: 301-496-2796; e-mail: Zachary.Lerner@nih.gov; DamianoD@cc.nih.gov; Thomas.Bulea@nih.gov).

U.S. Government work not protected by U.S. copyright
The conditions that accompany or contribute to crouch gait, such as muscle weakness, spasticity, and contracture, may adversely affect the efficacy of robotic assistance. Knowledge of the neuromuscular and kinematic adaptations to powered knee extension assistance in individuals with crouch gait is needed to optimize treatment strategies and evaluate the suitability of robotic devices for rehabilitation interventions. An increase in voluntary or involuntary muscle contractions of the knee flexors may be detrimental to device accommodation and rehabilitation outcomes. A reduction in muscle activity of the knee extensors may have negative implications for strength maintenance, but may also lead to a lower metabolic cost of walking and increased mobility.

The aim of this study was to evaluate how gait mechanics and muscle activity of the knee flexors and extensors are affected by powered knee extension assistance provided during stance and/or swing in individuals diagnosed with crouch gait caused by $\mathrm{CP}$.

\section{MATERIALS AND METHODS}

\section{A. Robotic Exoskeleton}

We developed a lower-extremity exoskeleton intended to treat crouch gait by providing powered knee extension assistance at different phases of the gait cycle, described in greater detail in [10]. The exoskeleton contains an adjustable, passive ankle joint, a knee joint incorporating a motor and transmission system, and sensors mounted to a custom molded thermoplastic orthotic brace (Fig. 1A). For this study, the ankle joint was set to allow free rotation and the actuated system was tethered to a power supply. A proportionalintegral-derivative motor control scheme was used to achieve a desired knee extensor torque at the exoskeleton's knee joint. When the torque was set to zero, the motor controller compensated for the inherent friction of the motor and transmission system, and allowed the joint to be articulated freely (i.e. frictionless).

Our motor controller implemented a finite state machine for determining when to provide the knee extension assistance by identifying the stance, early swing, and late swing phases of the gait cycle. Thresholds based on embedded foot sensor data were used to govern the transition between stance and swing states. Thresholds based on knee angle position and velocity obtained from the motor encoders were used to govern the transition between early (knee flexion) and late (knee extension) swing. We implemented three separate exoskeleton conditions that governed different modes of assistance (Fig. 1B); constant knee extension torque was provided during stance assist, swing assist, and combined stance and swing assist conditions. The torque setpoint data for each participant are presented in Table 1. 

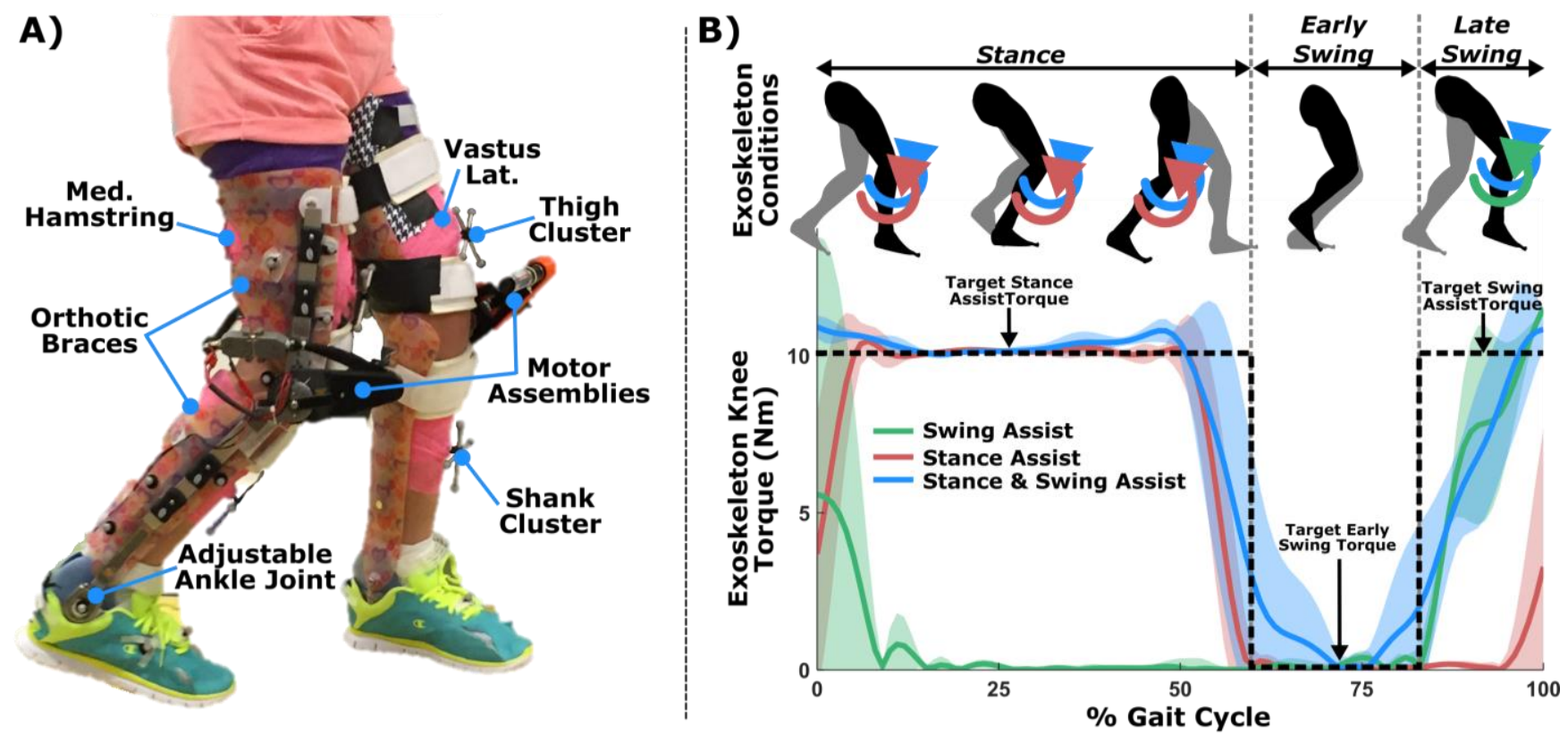

Figure 1. A) Subject \#2 walking with the robotic exoskeleton shown with labels indicating EMG electrode placements and exoskeleton components. B) Schematic depiction of the exoskeleton assistive conditions (top) and experimental torque data from a representative participant (subject \#4).

\section{B. Participants \& Experimental Protocol}

This study was approved by the Institutional Review Board at the National Institutes of Health (NIH). Four individuals (three females) with crouch gait caused by cerebral palsy were recruited for our study. Inclusion criteria included less than $5^{\circ}$ of knee flexion contracture and GMFCS $^{\text {a }}$ Level I-III. We obtained informed consent and assent from the parent and the participant, respectively. Participant information is presented in Table 1.

The participants completed 6 visits at the NIH over the course of the study. The first visit included assessment of leg flexibility, strength, and spasticity, an initial over-ground gait assessment, and lower-extremity casting for the creation of the orthotic braces. During the following 4 visits, participants acclimated to walking with the powered exoskeleton. The level of assistance (torque output) was incrementally adjusted, and verbally and visually evaluated to determine the optimal setting comfortably accommodated by each participant. The data presented in this study was from the $6^{\text {th }}$ visit, during which we collected experimental biomechanics data while the participants walked unassisted over ground in their every-day walking condition (baseline) and then with the exoskeleton under randomized stance, swing, and stance and swing assist conditions.

We recorded kinematic data using 10 motion capture cameras (Vicon Motion Systems, Oxford, UK). Individual retroreflective markers were placed on the feet, medial and lateral aspects of the ankles and knees, orthotic braces, pelvis, and trunk. Marker clusters, placed directly on each shank and thigh (Fig. 1A), were used to track the respective limb segments during the walking trials and establish consistent knee and ankle joint centers across baseline and exoskeleton conditions. Muscle activity data were collected from the vastus lateralis and medial hamstrings using a wireless EMG system recording at $1,000 \mathrm{~Hz}$ (Trigno Wireless, Delsys, Boston, MA). The orthotic braces were customized to accommodate electrode placement (Fig. 1A). Sensor data from the exoskeleton was synchronized with the motion capture system.

\section{Data \& Statistical Analysis}

We conducted an inverse kinematics analysis in Visual 3D (C-Motion, Gaithersburg, MD) to quantify lower-extremity joint angles from marker trajectories, which were low-pass filtered at $6 \mathrm{~Hz}$. EMG data were band-pass filtered at 15-380 $\mathrm{Hz}$, full-wave rectified, and low-pass filtered at $7 \mathrm{~Hz}$ to create a linear envelope [11]. EMG signals for each muscle were normalized to the peak value of the baseline condition. Numerical integration was used to calculate the area under the EMG - \% gait cycle curve during both the stance and swing portions of the gait cycle.

The experimental data were normalized to each gait cycle, and averaged across gait cycles for each condition. We used one-factor ANOVAs to determine how each condition affected the peak knee angles and muscle activity outcome measures for each participant. Post-hoc comparisons were made using the Tukey-Kramer method if a significant main event was found. Since some participants exhibited asymmetric gait deficits, we present the data from each participant's more affected limb, as determined by peak knee extension angle during stance.

TABLE I. PARTICIPANT INFORMATION AND EXOSKELETON PARAMETERS.

\begin{tabular}{ccccccccc}
\hline $\begin{array}{c}\text { Subject } \\
\text { (\#) }\end{array}$ & Age (yrs) & Height (m) & $\begin{array}{c}\text { Body Mass } \\
(\mathbf{k g})\end{array}$ & $\begin{array}{c}\text { GMFCS } \\
\text { Level }\end{array}$ & $\begin{array}{c}\text { Baseline } \\
\text { Condition }\end{array}$ & $\begin{array}{c}\text { Exoskeleton } \\
\text { Mass (kg) }\end{array}$ & $\begin{array}{c}\text { Stance Assist } \\
\text { Torque (Nm) }\end{array}$ & $\begin{array}{c}\text { Swing Assist } \\
\text { Torque (Nm) }\end{array}$ \\
\hline 1 & & & 20.0 & II & AFO & 3.16 & 3.5 & 3.5 \\
2 & 1.11 & 1.48 & 42.5 & II & AFO & 3.94 & 7 & 7 \\
3 & 11 & 1.56 & 40.8 & II & Shod & 3.88 & 10 & 10 \\
4 & 19 & 1.48 & 65.1 & III & Shod & 4.48 & 10 & 10 \\
\hline
\end{tabular}

${ }^{\mathrm{a}}$ GMFCS: Gross Motor Function Classification Scale. AFO: ankle-foot orthosis. Exoskeleton mass is the combined total for both limbs. 

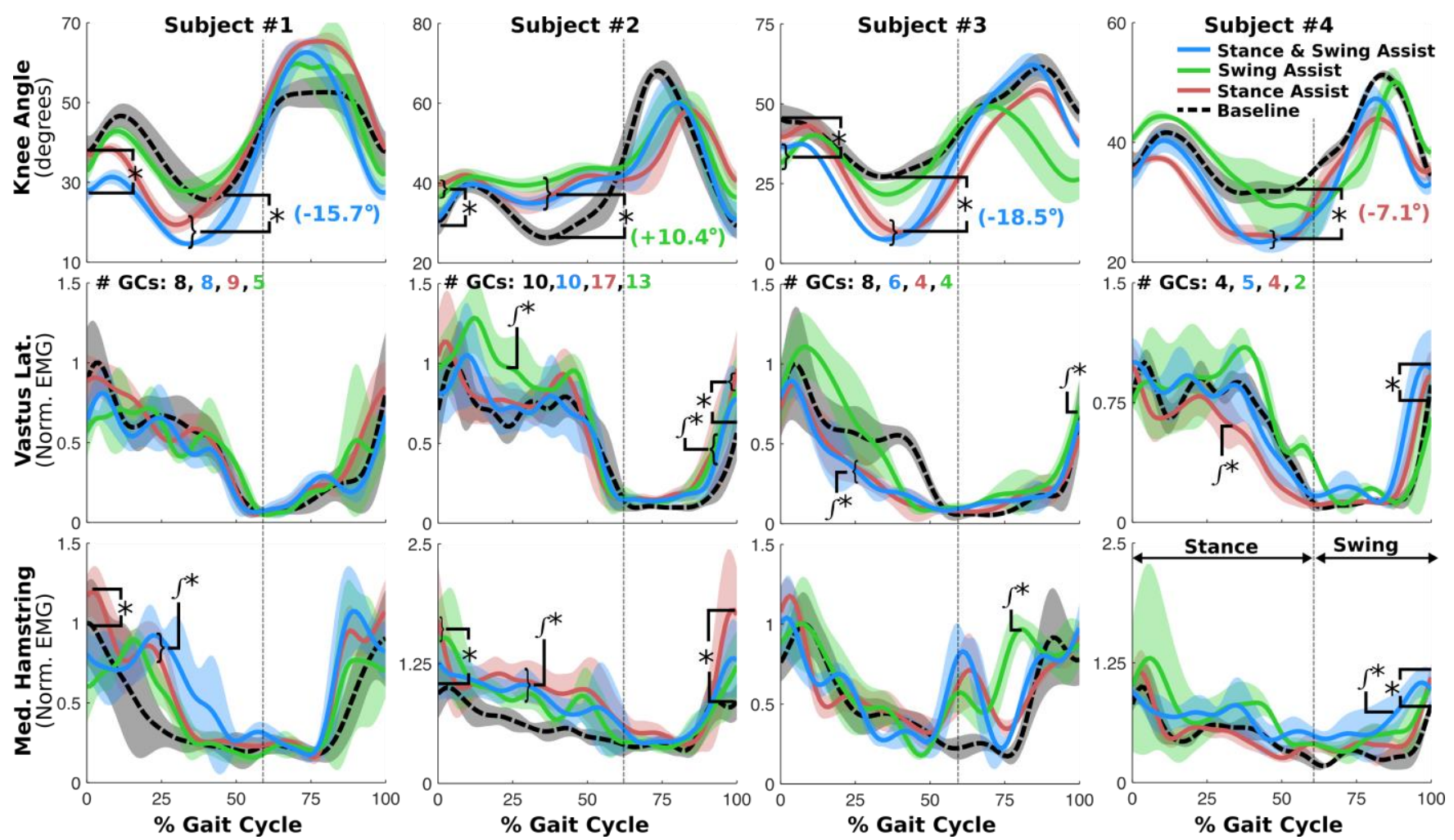

Figure 2. Knee flexion-extension angle (top row), vastus lateralis EMG (middle row), and medial hamstring EMG (bottom row) during baseline and exoskeleton assisted walking for each participant. Shading depicts \pm 1 standard deviation. * indicate a significant difference in peak knee angles or peak EMG. $\int^{*}$ indicates a significant difference in integrated EMG compared to baseline during either the stance or swing phases. Color coded numbers represent the number of gait cycles (\# GCs) available for analysis for each condition. The dashed vertical lines represent the average stance-swing transition.

\section{RESULTS}

All participants walked independently during baseline and with the exoskeleton across all conditions. We examined all available representative gait cycles for each participant and walking condition ( $7 \pm 4$, Fig. 2$)$.

\section{A. Knee Kinematics}

Maximum knee extension angle during stance increased by $13.8^{\circ}$ on average for subjects $\# 1, \# 3$, and $\# 4$ for conditions in which knee extension assistance was provided during the stance phase (Fig. 2), indicating the exoskeleton reduced crouch in these individuals. Conversely, maximum knee extension decreased by up to $10.4^{\circ}$ in subject $\# 2$. Knee extension at initial contact increased by $9.6^{\circ}$ in subject $\# 1$ during the stance $\&$ swing assist condition, and by $13.0^{\circ}$ and $8.5^{\circ}$ during the swing and stance \& swing conditions, respectively, in subject \#3.

\section{B. Muscle Activity}

Exoskeleton assistance altered EMG activity compared to baseline walking (Fig. 2, Table II). Peak vastus lateralis EMG activity was maintained across all assistive conditions for all four participants during stance. The integrated EMG for vastus lateralis during stance was reduced relative to baseline by $30 \%$ and $40 \%$ for subjects $\# 3$ and $\# 4$, respectively, for the stance assist condition. Peak and/or integrated swing phase vasti activity increased in subjects $\# 2$, \#3, and \#4. All participants exhibited an increase in peak and/or integrated hamstring EMG activity during either stance or swing, or both. The greatest increase $(70 \%)$ in integrated hamstring EMG occurred in subject \#2 during stance assist.

\section{DISCUSSION}

We present the preliminary kinematic and neuromuscular analysis of a robotic exoskeleton designed to treat crouch gait in four individuals with $\mathrm{CP}$. We observed a reduction in crouch, measured as an increase in knee extension during stance, in three of the four participants. Improvement was considered clinically significant when knee extension increased greater than 10 degrees, which is similar to results from hamstring lengthening surgery [12]. The variation across individuals in knee flexor and extensor muscle activity in response to powered assistance demonstrates challenges associated in treating this individualistic condition. However, all four subjects maintained peak knee extensor activity while walking with robotic assistance, indicating that they did not rely solely upon the exoskeleton to provide knee extension. This observation shows the potential for deployment of exoskeletons as a rehabilitation tool in this population.

Peak knee extensor activity was maintained in all participants and assistive conditions. The reduction in the integrated EMG for the vastus lateralis during walking with the exoskeleton under stance assistance for subjects \#3 and \#4 suggest a normalization of the activation pattern consistent with a more extended limb. While additional research is needed to evaluate the effect of powered extension assistance over a prolonged time period, the maintenance of peak knee extensor activity during short-term use suggests that some individuals with crouch gait may actively adopt an activation pattern that will result in an improvement in their posture, suggesting potential viability of the exoskeleton as a training device in this population. 
TABLE II. CHANGE IN MUSCLE ACTIVITY FOR VASTUS LATERALIS AND MED. HAMSTRINGS ACROSS THE ASSISTIVE CONDITIONS NORMALIZED TO BASELINE

\begin{tabular}{|c|c|c|c|c|c|c|c|c|c|c|c|c|c|c|}
\hline & \multicolumn{4}{|c|}{ Stance Assist } & \multicolumn{4}{|c|}{$\underline{\text { Swing Assist }}$} & \multicolumn{4}{|c|}{ Stance \& Swing Assist } \\
\hline & & & $\# 1$ & $\# 2$ & \#3 & $\# 4$ & $\# 1$ & $\# 2$ & $\# 3$ & $\# 4$ & $\# 1$ & $\# 2$ & \#3 & \#4 \\
\hline \multirow{4}{*}{ 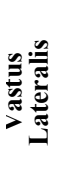 } & \multirow{2}{*}{ Stance } & Peak & $-0.1 \pm 0.1$ & $0.1 \pm 0.3$ & $-0.1 \pm 0.1$ & $0.0 \pm 0.1$ & $-0.2 \pm 0.1$ & $0.2 \pm 0.2$ & $0.2 \pm 0.1$ & $-0.1 \pm 0.3$ & $-0.2 \pm 0.2$ & $0.1 \pm 0.2$ & $-0.1 \pm 0.1$ & $0.1 \pm 0.1$ \\
\hline & & Integ & $0.0 \pm 0.1$ & $0.1 \pm 0.1$ & $-0.4 \pm 0.1$ & $-0.3 \pm 0.1$ & $-0.1 \pm 0.1$ & $0.3 \pm 0.1$ & $0.0 \pm 0.1$ & $0.1 \pm 0.1$ & $-0.1 \pm 0.1$ & $0.1 \pm 0.1$ & $-0.3 \pm 0.1$ & $0.0 \pm 0.1$ \\
\hline & \multirow{2}{*}{ Swing } & Peak & $0.1 \pm 0.2$ & $0.7 \pm 0.5$ & $-0.2 \pm 0.1$ & $0.1 \pm 0.2$ & $-0.2 \pm 0.4$ & $0.5 \pm 0.4$ & $0.1 \pm 0.3$ & $-0.2 \pm 0.4$ & $-0.2 \pm 0.2$ & $0.4 \pm 0.4$ & $0.0 \pm 0.2$ & $0.2 \pm 0.3$ \\
\hline & & Integ & $0.3 \pm 0.3$ & $0.6 \pm 0.4$ & $0.3 \pm 0.1$ & $0.4 \pm 0.2$ & $0.1 \pm 0.5$ & $0.6 \pm 0.5$ & $0.7 \pm 0.4$ & $0.0 \pm 0.6$ & $0.0 \pm 0.2$ & $0.5 \pm 0.5$ & $0.3 \pm 0.3$ & $1.2 \pm 0.5$ \\
\hline \multirow{4}{*}{ 㣢 } & \multirow{2}{*}{ Stance } & Peak & $0.1 \pm 0.1$ & $0.6 \pm 0.5$ & $0.1 \pm 0.1$ & $0.1 \pm 0.2$ & $-0.2 \pm 0.1$ & $0.5 \pm 0.5$ & $0.0 \pm 0.3$ & $0.3 \pm 0.9$ & $-0.1 \pm 0.1$ & $0.3 \pm 0.3$ & $0.0 \pm 0.3$ & $0.0 \pm 0.1$ \\
\hline & & Integ & $0.4 \pm 0.2$ & $0.7 \pm 0.2$ & $0.0 \pm 0.1$ & $0.0 \pm 0.1$ & $0.2 \pm 0.1$ & $0.5 \pm 0.1$ & $0.0 \pm 0.2$ & $0.5 \pm 0.5$ & $0.6 \pm 0.1$ & $0.5 \pm 0.2$ & $0.0 \pm 0.1$ & $0.3 \pm 0.2$ \\
\hline & \multirow{2}{*}{ Swing } & Peak & $0.2 \pm 0.2$ & $1.0 \pm 0.7$ & $0.0 \pm 0.1$ & $0.4 \pm 0.2$ & $-0.1 \pm 0.4$ & $0.3 \pm 0.3$ & $0.0 \pm 0.1$ & $0.2 \pm 0.1$ & $0.2 \pm 0.3$ & $0.4 \pm 0.4$ & $0.0 \pm 0.2$ & $0.3 \pm 0.2$ \\
\hline & & Integ & $0.4 \pm 0.1$ & $0.1 \pm 0.3$ & $0.3 \pm 0.1$ & $0.5 \pm 0.4$ & $0.1 \pm 0.5$ & $-0.1 \pm 0.3$ & $0.7 \pm 0.1$ & $0.5 \pm 0.8$ & $0.3 \pm 0.3$ & $0.1 \pm 0.3$ & $0.3 \pm 0.1$ & $1.4 \pm 0.6$ \\
\hline
\end{tabular}

Values are the normalized mean ( \pm standard deviation) change in EMG activity from baseline. Bold indicates a significant difference from the baseline condition $(p<0.05)$. Integ corresponds to the numerically integrated EMG.

An increase in antagonist muscle activity during walking with the exoskeleton may diminish rehabilitation outcomes. Our results suggest increased hamstring activity is associated with reduced kinematic improvements during stance and swing. For example, subject \#3 exhibited increased hamstring activity across stance phase in all exoskeleton conditions, which likely minimized improvement. This antagonistic activation may constitute an attempt by the participant to improve stability by maintaining a low center of gravity, and taking shorter steps, as confirmed in post-hoc analyses.

Our results may provide novel insights into motor control strategies for individuals with $\mathrm{CP}$ and enhance current understanding of the neuromuscular causes underlying crouch gait. Despite considerable assistance provided during the swing phase $(0.15-0.25 \mathrm{~N} / \mathrm{kg}$ body mass), there were limited improvements in knee extension at initial contact. The increase in hamstring EMG activity during swing assistance exhibited by several participants may suggest a control strategy that seeks to maintain a flexed posture at foot placement or a resistance to dynamic assistance in open chain movements. One idea for future research would be to investigate powered extension assistance with concurrent hamstring botulinum toxin injections.

Insights from this study may improve the design and control of robotic assistive devices in treating crouch gait. Providing assistance during the swing phase alone was insufficient to correct crouch across the entire gait cycle in this cohort. Compared to the swing assist condition, the stance and swing assist condition resulted in an increase in knee extension at initial contact for some of the participants. This suggests that a more extended stance limb may be critical for eliciting improved muscle function and knee extension during swing.

This study has several limitations. Our sample size was small, which may impact the generalizability of the findings. This is particularly true because the heterogeneous presentations of crouch gait make group-level analysis challenging. Still, we believe the results of this case series provide sufficient evidence to support continued research in this area. Another limitation was that the participants walked using the exoskeleton while it was tethered to a power-supply and control box. This was done to minimize participant burden during this physically demanding protocol and to more accurately isolate the effects of the different assist conditions. Using the exoskeleton without the tether would add $2-3 \mathrm{~kg}$ of mass to the user. The encouraging findings provide sufficient rationale for our continued development of lightweight embedded components and further investigation with a fully independent system.

\section{CONCLUSION}

The results of this study demonstrate the promise for powered knee extension assistance to supplement existing muscle function in individuals with crouch gait. Future research is needed to evaluate how muscle activity and neuromuscular control adapts during prolonged use.

\section{REFERENCES}

[1] E. Odding, M. E. Roebroeck, and H. J. Stam, "The epidemiology of cerebral palsy: incidence, impairments and risk factors," Disabil Rehabil, vol. 28, pp. 183-91, Feb 2006.

[2] T. A. Wren, S. Rethlefsen, and R. M. Kay, "Prevalence of specific gait abnormalities in children with cerebral palsy: influence of cerebral palsy subtype, age, and previous surgery," J Pediatr Orthop, vol. 25 , pp. 79-83, Jan 2005.

[3] R. Jahnsen, L. Villien, G. Aamodt, J. K. Stanghelle, and I. Holm, "Musculoskeletal pain in adults with cerebral palsy compared with the general population," J Rehabil Med, vol. 36, pp. 78-84, Mar 2004.

[4] A. Opheim, R. Jahnsen, E. Olsson, and J. Stanghelle, "Walking function, pain, and fatigue in adults with cerebral palsy: a 7-year followup study," Dev Med Child Neurol, vol. 51, pp 381-8, May 2009.

[5] J. Rose, J. G. Gamble, A. Burgos, J. Medeiros, and W. L. Haskell, "Energy expenditure index of walking for normal children and for children with cerebral palsy," Dev Med Child Neurol, vol. 32, pp. 33340, Apr 1990.

[6] C. De Mattos, K. Patrick Do, R. Pierce, J. Feng, M. Aiona, and M. Sussman, "Comparison of hamstring transfer with hamstring lengthening in ambulatory children with cerebral palsy: further follow-up," J Child Orthop, vol. 8, pp. 513-20, Dec 2014.

[7] D. L. Damiano, C. L. Vaughan, and M. F. Abel, "Muscle response to heavy resistance exercise in children with spastic cerebral palsy," Dev Med Child Neurol, vol. 37, pp. 731-9, Aug 1995.

[8] M. A. Brehm, J. Harlaar, and M. Schwartz, "Effect of ankle-foot orthoses on walking efficiency and gait in children with cerebral palsy," J Rehabil Med, vol. 40, pp. 529-34, Jul 2008.

[9] M. Bottos and C. Gericke, "Ambulatory capacity in cerebral palsy: prognostic criteria and consequences for intervention," Dev Med Child Neurol, vol. 45, pp. 786-90, Nov 2003.

[10] Z. Lerner, D. Damiano, and T. Bulea, " A Robotic Exoskeleton for Treatment of Crouch Gait in Children with Cerebral Palsy: Design and Initial Application," IEEE Trans Neural Syst Rehabil Eng, Accepted.

[11] D. A. Winter, "The Biomechanics and Motor Control of Human Gait," University of Waterloo Press, Waterloo, Ontario, 1987.

[12] J. Thometz, S. Simon, and R. Rosenthal, "The effect on gait of lengthening of the medial hamstrings in cerebral palsy," J Bone Joint Surg Am, vol. 71, pp. 345-53, Mar 1989. 